\title{
Making MIC Work for You: Utilizing PK and PD Parameters
}

\section{Dharmendra Sharma ${ }^{1}$ and Ashok Rattan ${ }^{2 *}$}

${ }^{1}$ Associate Professor, Department of Basic Medical Sciences, College of Medicine at

Shaqra, Shaqra University, Kingdom of Saudi Arabia

${ }^{2}$ Adviser, Laboratory Operations, Pathkind Labs, Pathkind Diagnostics Pvt Ltd., Gurgaon,

Haryana, India

*Corresponding Author: Ashok Rattan, Adviser, Laboratory Operations, Pathkind

Labs, Pathkind Diagnostics Pvt Ltd., Gurgaon, Haryana, India.
Received: July 20, 2020

Published: September 16, 2020

(C) All rights are reserved by Dharmendra

Sharma and Ashok Rattan.

\section{Abstract}

MIC or MBC has been used as indicator of antibiotic potency towards a pathogen and as surrogate marker for clinical efficacy when the selected antibiotic is used in the patient, but these parameters are static measures and do not adequately reflect the dynamic process that takes place in a patient. To better predict the clinical efficacy of the drug we need to take into consideration the pharmacokinetics (what the host does to the drug) and the pharmacodynamics (what the drug does to the bug) and to obtain the three PK/PD parameters each of which depends upon the MIC value obtained but better predictive the clinical efficacy of the selected antibiotic.

Keywords: Minimum Inhibitory Concentration (MIC); Minimum Bactericidal Concentration (MBC); PK and PD Parameters

\section{Introduction}

Microbiologists consider Minimum Inhibitory Concentration (MIC) values as good parameters of antibiotic potency but when they report MIC values to the clinicians most find it difficult to comprehend and they request for classifying the results as either Sensitive or Resistance. MIC or for that matters Minimum Bactericidal Concentration (MBC) values do not provide information on the rate of bactericidal activity and MIC values do not provide any information on the persistent effects of antibacterial agents. Shah., et al. [1] were the first investigators to propose that antibacterials could be divided into different groups on the basis of their patterns of bactericidal activity. The first pattern is characterized by concentration dependent killing, the higher the concentration of the drug, the greater the rate and extent of bactericidal activity. This pattern was observed in vitro in kinetic kill experiments, in vivo with infected mice and in patients during clinical trials and is best exhibited by aminoglycosides and fluoroquinolones (Figure 1).

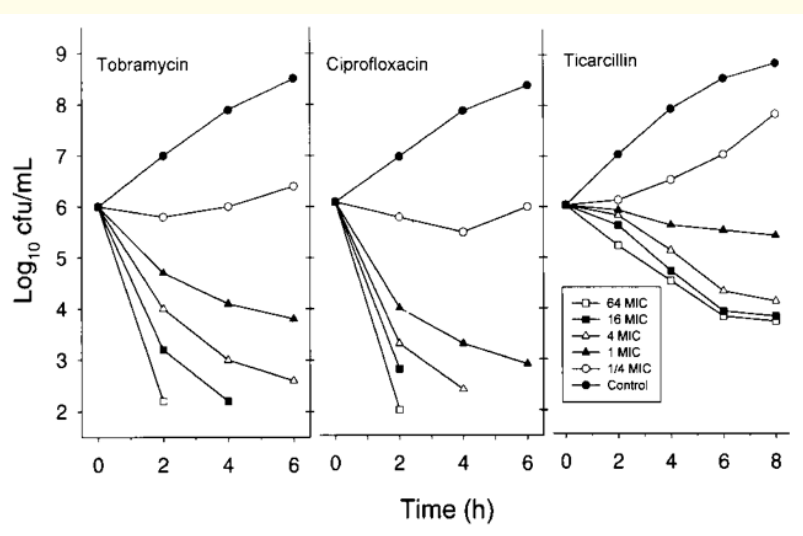

Figure 1: Kinetic kill of $E$. coli with different antibiotics at various MIC concentrations, tobramycin and ciprofloxacin show concentration dependent killing. 
In contrast, the second pattern is characterized by minimal concentration dependent killing and once the levels have exceeded about 4 times the MIC, there is no improvement by increasing the concentration of the drug. The extent of killing is largely dependent on the time of exposure. This is characteristically seen with beta lactams, cephalosporins, vancomycin, clindamycin and macrolides. Another problem with MIC is that it is static phenomenon representing the end point of a dynamic interaction and is not appropriate for predicting the final clinical response without taking into consideration the pharmacokinetics (what the body does to the drug) and pharmacodynamics (what the drug does to the bug) [2].

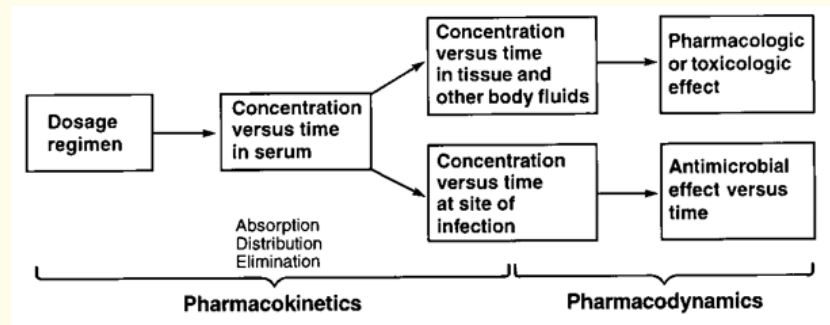

Figure 2: Time course of drug levels in the body with pharmacokinetics and pharmacodynamics.

The three pharmacokinetic parameters of significance are: $\mathrm{C}_{\max }$, AUC and $t_{1 / 2}$ which together with MIC are used to obtain the PK PD parameters of successful antibiotic use as $\mathrm{C}_{\max } / \mathrm{MIC}>10$ or AUC/ MIC $>100$ or time over MIC of $>40 \%$ of antibiotic dosing. In other words, for concentration dependent drugs, as it is either $\mathrm{C}_{\max } / \mathrm{MIC}$ or AUC/MIC that needs to over 10 or 100 respectively, these drugs need to be administered as a single large dose, while for drugs like beta lactams or cephalosporins it is the \% of time that the drug was over MIC, minimum required for efficacy being $40 \%$ of dosing interval, so more frequent dosing would be ideal [3].

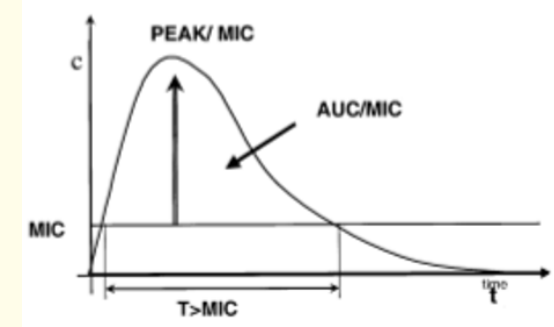

Figure 3: The different pharmacokinetics parameters after drug administration.
It is difficult to memorise $\mathrm{C}_{\text {max }}$ AUC or $t_{1 / 2}$ of different drugs, though these values are available in the product inserts approved by FDA as these data are obtained by the drug manufacturer during clinical development of the antibiotic. To aid the clinician and laboratory scientist, we have used these values and entered them in an excel software and used the formulae explained below.

Most of the drugs have been divided into either concentration dependent or Time dependent groups as under.

Concentration dependent drugs:

- Aminoglycosides

- Fluoroquinolones

- Metronidazole

- Daptomycin

- $\quad$ Ketolides

- Azithromycin

- Streptogramin

- Glycopeptides

- Amphotericin

- Fluconazole

Time dependent drugs:

- Penicillin

- Cephalosporins

- Carbapenems

- Monobactam

- Macrolides

- Clindamycin

- Oxazolidinones

- Glycylcyclines

- Flucytosine

PK/PD parameters predictive of success

For concentration dependent drugs

- $\mathrm{Cmax} / \mathrm{MIC}>10$.

- $\quad \mathrm{AUC} / \mathrm{MIC}>100$.

For time dependent and concentration independent drugs

- $\mathrm{T} 1 / 2>40 \%$ of dosing interval. 


\section{Methodology and Results}

\section{Methods of calculation}

\section{Time over MIC}

As we know that in a single compartment model the antibiotic concentration - time curve is an exponentially falling curve, in which over a period of time a defined fraction of the antibiotic concentration falls. The commonly used time period is $\mathrm{T}_{1 / 2}$ during which the antibiotic concentration falls to the half of its concentration at the beginning.

The relationship of serum concentration to the beginning concentration is usually represented by the following formula [4]:

$\mathrm{C}_{\mathrm{t}}=\mathrm{C}_{0} \times \mathrm{e}^{-\mathrm{Kt}}$

Where $\mathrm{C}_{0}=$ Serum concentration at the beginning

$\mathrm{C}_{\mathrm{t}}=$ Serum concentration after the time " $\mathrm{t}$ "

$\mathrm{K}=$ Elimination rate constant

$\mathrm{t}=$ Time

$\mathrm{e}=$ Base of the natural logarithm.

We found an easier way to calculate the time over MIC, which is in other words the time required for the concentration to fall from $\mathrm{C}_{\text {max }}$ to the level of MIC in case of intravenous administration of the drug.

To begin with the calculation we need the value of $\mathrm{C}_{\max }$ for a particular dose, value of $\mathrm{T}_{1 / 2}$, (both of these values for a particular antibiotic are available in the text books and on FDA website) and the value of MIC of that antibiotic for the clinical isolate.

Suppose that the serum antibiotic concentration will take " $n$ " number of half-lives to fall from the level of $\mathrm{C}_{\max }$ to MIC. Then it can be represented by the following formula: $\frac{c_{\max }}{2^{n}}=M I C$

Which means that dividing the $\mathrm{C}_{\max }$ value by 2 for " $\mathrm{n}$ " times gives the value of MIC. This equation can also be written as: $\frac{c_{\max }}{M I C}=2^{n}$

From this we can find the value of " $n$ " (number of half-lives) by taking the logarithm of both sides of the equation on the base $2\left(\log _{2}\right)$.

$\log _{2}\left(\frac{C_{\max }}{M I C}\right)=\log _{2}\left(2^{\mathrm{n}}\right)$

Or $\log _{2}\left(\frac{C_{\max }}{M I C}\right)=\mathrm{n}$
Here " $n$ " represents the number of half-lives required over which the serum concentration of antibiotic will from the level of $\mathrm{C}_{\max }$ to MIC. Now we can easily find the value of time over MIC by multiplying the value of " $n$ " with the value of $\mathrm{T}_{1 / 2}$.

Time over $\mathrm{MIC}=\mathrm{n} \times \mathrm{T}_{1 / 2}$

These calculations can be done very easily, using the Log function in the Microsoft Excel.

Step 1 - Take the ratio of $\mathrm{C}_{\max }$ and $\operatorname{MIC}\left(\frac{c_{\max }}{\text { MIC }}\right)$

Step 2 - Use the LOG function to find the Log of the value obtained in step 1 , by selecting the base as 2 .

Step 3 - Multiply the result of the step 2 with the value of $\mathrm{T}_{1 / 2}$. The result is time over MIC.

\section{Area under curve (AUC)}

There are two methods which are commonly used to calculate the AUC. One is the trapezoidal method and the other one is by using the integral calculus [4].

We began with the trapezoidal method to calculate AUC for the single compartment model of intravenously administered antibiotics, and used $\mathrm{C}_{\max }$ as initial concentration and $\mathrm{T}_{1 / 2}$ as the measure of point in time at which the value of another serum concentration is available (half of the preceding one).

The AUC for the duration of first half life $\left(\mathrm{AUC}_{1}\right)$ with the initial serum concentration of antibiotic is $\mathrm{C}_{\max }$ can be represented as:

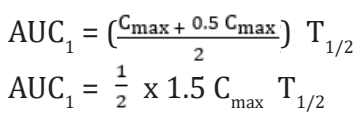

For the AUC during second half-life $\left(\mathrm{AUC}_{2}\right)$, the beginning serum concentration will be half of the $\mathrm{C}_{\max }$ and as a result the value of $\mathrm{AUC}_{2}$ will also be half of the AUC ${ }_{1}$. In the same way the AUC for all the subsequent half-lives will exponentially decrease by a factor of $1 / 2$.

Keeping in mind these facts if we want to find AUC for the duration ranging from $\mathrm{C}_{\max }$ to a certain number of half-lives (n), we can sum the values of AUCs during all these half-lives, which can be represented as:

$\mathrm{AUC}_{(0-\mathrm{n})}=\mathrm{AUC}_{1}+\mathrm{AUC}_{2}+\ldots \ldots \ldots \ldots \ldots+\mathrm{AUC}_{\mathrm{n}}$ 


$$
\begin{aligned}
& \operatorname{AUC}_{(0-\mathrm{n})}=\left(\frac{1}{2} \times 1.5 \mathrm{C}_{\max } \times \mathrm{T}_{1 / 2}\right)+\left(\frac{1}{2^{2}} \times 1.5 \mathrm{C}_{\max } \times \mathrm{T}_{1 / 2}\right)+\ldots \ldots+\left(\frac{1}{2^{n}} \times 1.5 \mathrm{C}_{\max } \times \mathrm{T}_{1 / 2}\right) \\
& \operatorname{AUC}_{(0-\mathrm{n})}=1.5 \mathrm{C}_{\text {max }} \mathrm{T}_{1 / 2}\left(\frac{1}{2}+\frac{1}{2^{2}}+\frac{1}{2^{3}}+\ldots \ldots \ldots \ldots \ldots+\frac{1}{2^{n}}\right)
\end{aligned}
$$

After simplification

$\operatorname{AUC}_{(0-\mathrm{n})}=1.5 \mathrm{C}_{\max } \mathrm{T}_{1 / 2}\left(\frac{2^{\mathrm{n}}-1}{2^{\mathrm{n}}}\right)$

Now if we want to calculate the $A U C_{\infty}$ then:

$$
\begin{aligned}
& A U C_{\infty}=1.5 \mathrm{C}_{\max } \times \mathrm{T}_{1 / 2} \times\left(\frac{2^{\infty}-1}{2^{\infty}}\right) \\
& =1.5 \mathrm{C}_{\max } \times \mathrm{T}_{1 / 2} \times 1 \text { because }\left(2^{\infty}-1\right) \approx 2^{\infty} \\
& =1.5 \mathrm{C}_{\max } \times \mathrm{T}_{1 / 2}
\end{aligned}
$$

So, the value of $A U C_{\infty}$ is equal to $1.5 \mathrm{Cmax} . \mathrm{T} 1 / 2$ by this method.

Later we explored the standard text book formula derived by integral calculus for finding $A U C_{\infty}[4]$ :

$$
\mathrm{AUC}_{\infty}=\frac{\mathrm{C}_{\mathrm{max}}}{\mathrm{K}_{\mathrm{el}}}
$$

Where Kel is elimination constant, which is equal to " $\frac{\operatorname{In} 2}{T_{1 / 2}}$, where In2 is the natural lnarithm of 2 . If we replace the "Kel" in the above formula with " $\frac{\operatorname{In} 2}{\mathrm{~T}_{1 / 2}}$, then:

$$
\begin{aligned}
& \mathrm{AUC}_{\infty}=\frac{\mathrm{C}_{\max }}{\operatorname{In} 2 / \mathrm{T}_{1 / 2}} \\
& =\frac{\mathrm{C}_{\max } \times \mathrm{T}_{1 / 2}}{\operatorname{In2}} \\
& =\frac{\mathrm{C}_{\max } \times \mathrm{T}_{1 / 2}}{0.693} \text { (the value of } I n 2 \text { is a constant equal to } 0.693 \text { ) } \\
& =1.44 \mathrm{C}_{\max } \cdot \mathrm{T}_{1 / 2}
\end{aligned}
$$

So, the value of $A U C_{\infty}$ by integral calculus is equal to $1.44 \mathrm{C}_{\max }$. $\mathrm{T}_{1 / 2}$ which is slightly less than the trapezoidal method and more accurate. As shown in the figure.

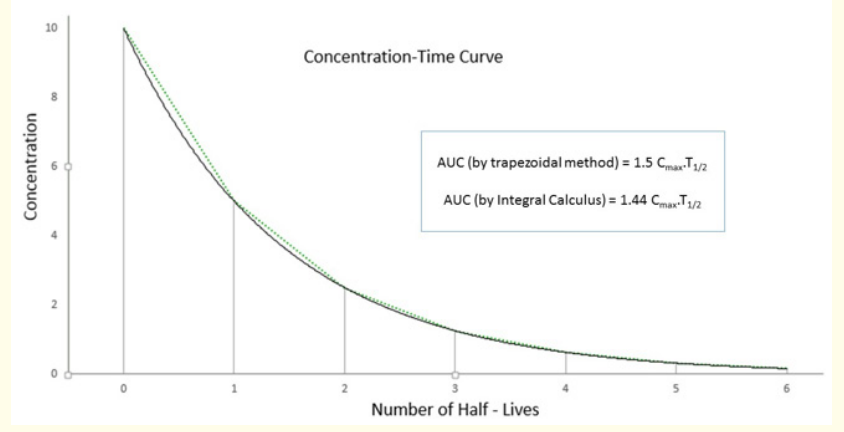

Figure 4: Calculating AUC of the drug.
This formula is simple and can also be easily incorporated in the Excel sheet for automated calculation of AUC.

Now to find out AUC for a particular duration " $t$ " after a single dose, the calculations can be done as follows:

$\mathrm{AUC}_{(0-\mathrm{t})}=\left(A U C_{\infty}\right.$ after the concentration at the beginning $)-$ $\left(A U C_{\infty}\right.$ after the concentration at time " $\mathrm{t}$ ") $=\left(1.44 \mathrm{C}_{\max } \mathrm{T}_{1 / 2}\right)-(1.44$ $\mathrm{C}_{\mathrm{t}} \cdot \mathrm{T}_{1 / 2}$ )

Or $\mathrm{AUC}_{(0-\mathrm{t})}=\left(\mathrm{C}_{\max }-\mathrm{C}_{\mathrm{t}}\right)\left(1.44 \mathrm{~T}_{1 / 2}\right)$

The standard formula for finding the value of " $\mathrm{C}_{t}$ " is [4]:

$\mathrm{C}_{\mathrm{t}}=\mathrm{C}_{0} \times \mathrm{e}^{-\mathrm{Kt}}$

We have found a simpler formula to calculate the same, and the result of both these formulae are exactly the same:

$C_{t}=\frac{C_{0}}{2^{n}}$

Where " $n$ " is equal to ( $t / T_{1 / 2}$ ), the number of half-lives passed after the $\mathrm{C}_{0}$.

By using these formulae we can calculate the 24 hour AUC for a single antibiotic dose. We can calculate AUC/MIC value by summing up AUCs of all the doses administered during 24h and then by dividing the resultant sum with MIC.

\section{Excel based program for these calculations}

We have made an Excel based program to automate these calculations. One would get three screens in this program. We need to enable Macros for the software to run. Please do not change anything on the formula page, while you are free to add more drugs and values in the database. The home page would appear as under. By clicking on the window you can select one of the antibiotics from the list, then enter the MIC value and dose interval and press calculate to obtain PK PD parameters.

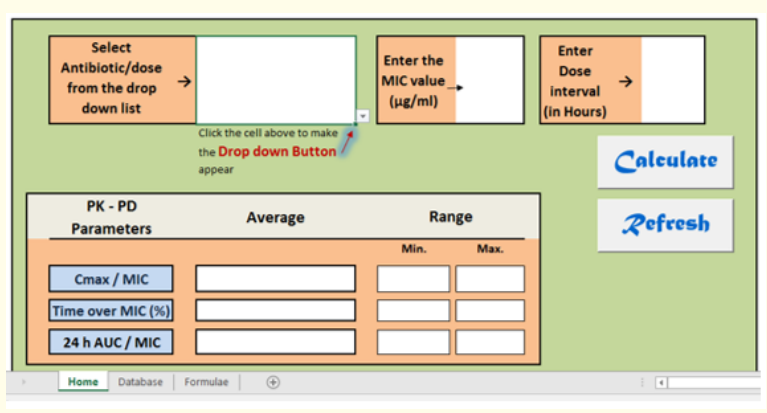

Figure 5: The home screen in excel. 


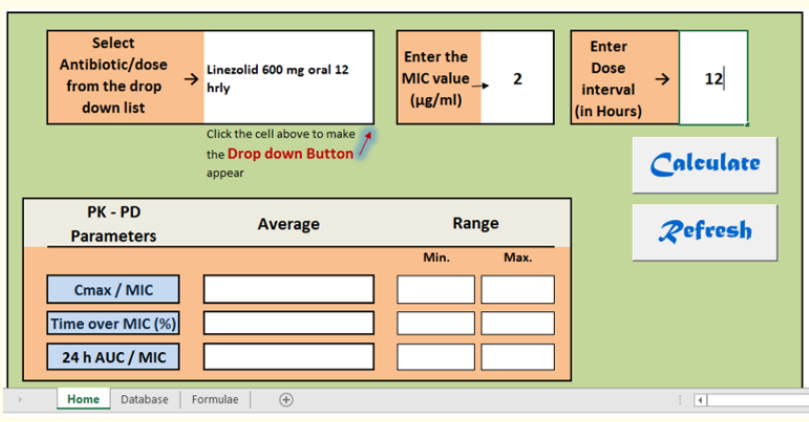

Figure 6: If we wish to use Linezolid $600 \mathrm{mg}$ twice a day against a bacteria with MIC of $2 \mathrm{ug} / \mathrm{ml}$.

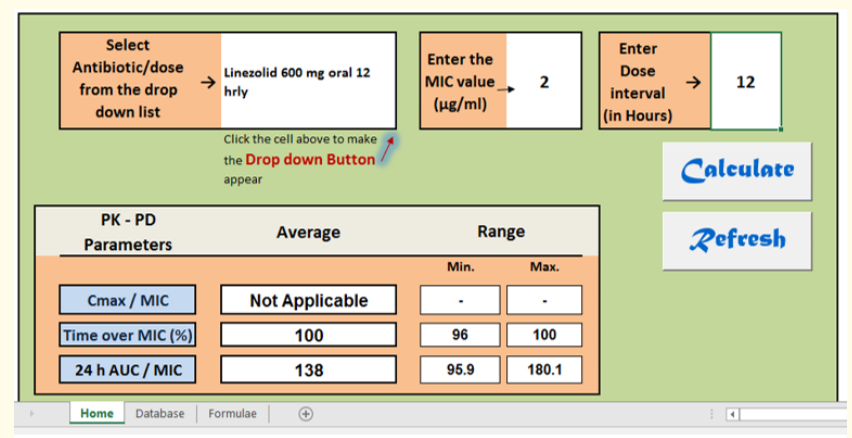

Figure 7: $\mathrm{T} 1 / 2$ should be $>40 \%$ of doing interval, in this case the drug will be effective against this bacteria.

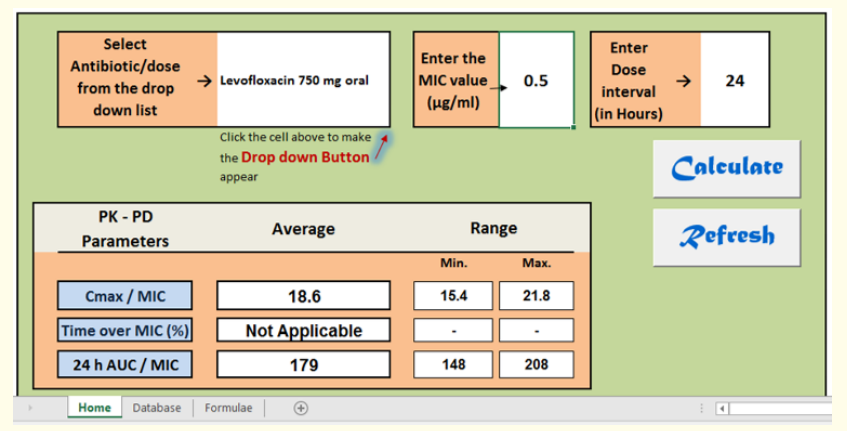

Figure 8: If Levofloxacin $750 \mathrm{mg}$ once a day is to be used against a bacteria with MIC of $0.5 \mathrm{mg} / \mathrm{ml}$ the Cmax/MIC is in the effective range.

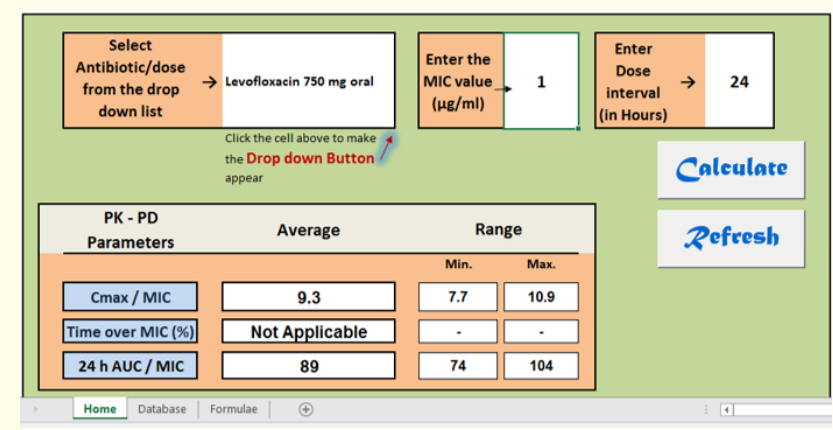

Figure 9: However Levofloxacin $750 \mathrm{mg} / \mathrm{ml}$ once daily may not succeed if the MIC of the bacteria is $1 \mathrm{mg} / \mathrm{ml}$ and the Levofloxacin $\mathrm{PK}$ is not at its best, so chances of failure exits.

\section{Discussion}

The available methods for calculation for PK-PD parameters depend on many constants and terminologies which are not familiar to common practitioners. Our work simplifies the calculations and makes it user friendly. Our method of calculation has been converted into an automated Excel program for immediate calculation of PK-PD parameters.

For use of the software, once MIC value has been obtained in the laboratory, input is required regarding the drug to be used, the dose and frequency that you would like to administer it, once you input the MIC, the program calculates the relevant parameters and gives out the $\mathrm{C}_{\max } / \mathrm{MIC}$ and AUC/MIC for concentration dependent drugs or $t_{1 / 2} /$ MIC for time dependent drugs as the case maybe. Success with the selected antibiotic can be predicted if the relevant PK PD threshold is crossed.

\section{Conclusion}

Our software allows the laboratory personnel or clinician to easily predict whether the antibiotic selected is the most appropriate by taking both the MIC as well as PK PD parameters into consideration at the selected dose and frequency.

We would be happy to share the software with interested scientists and clinicians. 


\section{Bibliography}

1. Shah PM., et al. "Bactericidal dosie-activity relationships with E. coli, K. pneumoniae and Staph. aureus (author's transl)". Deutsche Medizinische Wochenschrift 101 (1976): 325-328.

2. Craig WA. "Pharmacokinetic/pharmacodynamic parameters: rationale for antibacterial dosing of mice and men". Clinical Infectious Diseases 26.1 (1998): 1-12.

3. Drusano GL. "Pharmacokinetics and pharmacodynamics of antimicrobials". Clinical Infectious Diseases 45 (2007): S89S95.

4. Jambhekar SS and Breen PJ. "Basic Pharmacokinetics: $2^{\text {nd }}$ edition”. (Pharmaceutical press, London, UK) (2012): 55-77.

\section{Assets from publication with us}

- Prompt Acknowledgement after receiving the article

- Thorough Double blinded peer review

- Rapid Publication

- Issue of Publication Certificate

- High visibility of your Published work

Website: https://www.actascientific.com/

Submit Article: https://www.actascientific.com/submission.php

Email us: editor@actascientific.com

Contact us: +919182824667 